Case Reports in
Gastroenterology
Case Rep Gastroenterol 2020;14:586-592

DOI: 10.1159/000509953

Published online: November 2, 2020

(c) 2020 The Author(s)

Published by S. Karger AG, Base

www.karger.com/crg

This article is licensed under the Creative Commons Attribution-NonCommercial 4.0 International License (CC BY-NC) (http://www.karger.com/Services/OpenAccessLicense).

Usage and distribution for commercial purposes requires written permission.

\title{
Pembrolizumab-Induced Delayed-Onset Hepatitis
}

\author{
Kensuke Kanaoka ${ }^{a} \quad$ Kazunori Moriizumi ${ }^{a}$ Hideyasu Okada ${ }^{a}$ \\ Kiyoshi Iwahashi $^{\mathrm{b}}$ Hiromi Tsuji $^{\mathrm{c}}$ Hironao Yasuoka ${ }^{c}$ Seigo Minami ${ }^{a}$ \\ aDepartment of Respiratory Medicine, Osaka Police Hospital, Osaka, Japan; \\ ${ }^{b}$ Department of Gastroenterology, Osaka Police Hospital, Osaka, Japan; \\ 'Department of Pathology, Osaka Police Hospital, Osaka, Japan
}

\section{Keywords}

Pembrolizumab · Immune-related adverse events · Hepatitis · CD3-positive lymphocyte · CD8-positive lymphocyte

\begin{abstract}
Hepatitis is one of the serious immune-related adverse events (irAEs). However, delayed-onset hepatitis induced by immune-checkpoint inhibitors (ICls) is rare, and the histopathological features remain to be clarified. A 65-year-old woman with advanced lung adenocarcinoma in the right upper lobe (cT4N3M1C, c-stage IVB) received four courses of pembrolizumab. Her hepatic and biliary tract enzyme levels started increasing 2 months after the final administration of pembrolizumab, and the elevated levels of these enzymes prolonged. Liver biopsy revealed panlobular infiltration of inflammatory cells, and most of the infiltrating inflammatory cells were lymphocytes; however, there were a small number of neutrophils, eosinophils, and plasma cells. There was no confluent necrosis. Furthermore, immunohistochemical analyses proved that infiltrating lymphocytes were predominantly $C D 3$-positive $(C D 3+)$ and $C D 8+$, and few CD20+ and CD4+ lymphocytes were observed. Based on these findings, she was diagnosed with a case of hepatitis as an irAE. Administration of prednisolone $(0.5 \mathrm{mg} / \mathrm{kg} / \mathrm{day})$ as well as the addition of azathioprine failed to suppress the deterioration. However, an increase in the dose of prednisolone (up to $1 \mathrm{mg} / \mathrm{kg} /$ day) enabled us to control hepatitis. This case showed that hepatitis can occur even after discontinuation of $\mathrm{ICls}$, and that liver biopsy may be useful in the diagnosis. Clinicians should not hesitate to perform liver biopsy for confirmation of the diagnosis.

(C) 2020 The Author(s)

Published by S. Karger AG, Basel
\end{abstract}




\section{Case Reports in Gastroenterology}

Case Rep Gastroenterol 2020;14:586-592 DOI: 10.1159/000509953

\section{Introduction}

Pembrolizumab has been reported to improve the overall survival of patients with advanced non-small cell lung carcinoma with a programmed cell death ligand 1 (PD-L1) tumor proportion score of $1 \%$ or greater, compared with platinum-based chemotherapy [1]. Among the various immune-related adverse events (irAEs) induced by programmed cell death 1 (PD1) or PD-L1 inhibitors, hepatitis is a serious adverse event [2]. Hepatitis has been reported in $0.85 \%$ of patients treated with PD-1 or PD-L1 inhibitors, with grade 3 and higher severity in $0.43 \%$ [2]. A study reported that the median duration between the initiation of PD-1/PD-L1 inhibitor therapy and the onset of hepatitis was 14 weeks [3]. In general, delayed-onset hepatitis induced by immune-checkpoint inhibitors (ICIs) is uncommon.

The typical pathological pattern of hepatitis as an irAE is panlobular inflammation with spotty necrosis, and confluent necrosis is less common. Most of the infiltrating inflammatory cells are lymphocytes, and few eosinophils, neutrophils, and plasma cells may be observed [4, 5]. Furthermore, immunostaining has demonstrated low CD20+/CD3+ and CD4+/CD8+ cell ratios of the infiltrating lymphocytes. These findings are useful to distinguish hepatitis as an irAE from autoimmune hepatitis or idiosyncratic drug-induced liver injury [5]. To the best of our knowledge, histopathological analysis of delayed-onset hepatitis has never been reported.

The guideline published by the American Society of Clinical Oncology (ASCO) recommends corticosteroids as the first-line treatment for hepatitis as an irAE of grade 2 or higher [6]. The recommended corticosteroid dose differs depending the severity of hepatitis, which is determined based on the level of aspartate aminotransferase (AST), alanine transaminase (ALT), and bilirubin. Briefly, the doses are $0.5-1 \mathrm{mg} / \mathrm{kg} /$ day of prednisone or equivalent for grade 2 hepatitis, 1-2 mg/kg of methylprednisolone or equivalent for grade 3 hepatitis, and 2 $\mathrm{mg} / \mathrm{kg}$ of methylprednisolone or equivalent for grade 4 hepatitis. Corticosteroid therapy leads to normalization or improvement of serum liver enzymes in most patients. A study reported that median time from initiation of corticosteroids to resolution was approximately 8 weeks [7]. Furthermore, the ASCO recommends additional treatment with mycophenolate mofetil or azathioprine in cases where corticosteroids are not effective [6].

Herein, we report a case of hepatitis as an irAE that developed 2 months after the final administration of pembrolizumab and was subsequently confirmed histopathologically by liver biopsy.

\section{Case Report}

A 65-year-old woman visited our hospital after detection of an abnormal shadow on chest $\mathrm{X}$-ray. She was diagnosed with a lung adenocarcinoma in the right upper lobe (cT4N3M1c, cstage IVB), with distant metastases to the brain, adrenal glands, subcutaneous tissue at the back, and ileocecal lymph node. However, metastasis to the liver was not detected. The tumor did not harbor mutant epidermal growth factor receptor, rearranged anaplastic lymphoma kinase fusion oncogene, and mutant c-ros oncogene, but showed high tumor proportion score of PD-L1 (70-80\%). As the first-line regimen, we initiated pembrolizumab monotherapy at a dose of 200 mg every 3 weeks. We postponed the second course of pembrolizumab by 4 weeks due to $\gamma$-knife irradiation of brain metastases and surgical resection of the cerebellar metastasis. We also postponed the third course by 3 weeks due to grade 3 increase in the level of alkaline phosphatase (ALP) and gamma-glutamyl transpeptidase ( $\gamma$-GTP), which occurred 68 days after the initiation of pembrolizumab therapy. Pembrolizumab was restarted after a 


\section{Case Reports in Gastroenterology}

Case Rep Gastroenterol 2020;14:586-592 DOI: $10.1159 / 000509953$

c) 2020 The Author(s). Published by S. Karger AG, Basel www.karger.com/crg

Kanaoka et al.: Pembrolizumab-Induced Delayed-Onset Hepatitis

spontaneous decrease in the ALP and $\gamma$-GTP levels. After a total of four courses, we confirmed progression of the primary lesion and ileocecal lymph node metastasis. We switched the treatment to cytotoxic chemotherapy with carboplatin, pemetrexed, and bevacizumab. After two courses of this regimen ( 169 days after initiation and 59 days after the final administration of pembrolizumab), an increase in AST, ALT, ALP, and $\gamma$-GTP levels was observed. Subsequently, the level of these enzymes reached grades 2, 2, 3, and 4, respectively (Fig. 1). Furthermore, serum bilirubin levels increased to the grade 3 range $(3.8 \mathrm{mg} / \mathrm{dL})$; however, it spontaneously decreased to the grade 2 range. We stopped chemotherapy; however, the levels of these enzymes remained elevated. The patient did not complain of any symptoms and did not present with fever. Serum levels of immunoglobulin M (IgM), IgG, and IgG4 were within the normal range. Antinuclear, anti-mitochondrial, cytomegalovirus-IgM, and hepatitis $\mathrm{C}$ virus antibodies were negative, and the Epstein-Barr nuclear antigen was undeterminable. Virus capsid antigen-IgG was 160-fold, which was not significantly elevated to suspect chronic active EpsteinBarr virus infection. Although anti-hepatitis B envelope and core antibodies were positive, hepatitis B viral DNA was not detected by real-time polymerase chain reaction. Computed tomography showed mild dilation of the intrahepatic and common bile ducts; however, neither cholecystitis nor liver metastasis was detected (Fig. 2). The patient discontinued all prescribed medicines, namely, codeine phosphate, dextromethorphan, lansoprazole, bilastine, carbocysteine, ambroxol hydrochloride, and retinol/calciferol combination drug. However, the elevated levels of AST, ALT, ALP, and $\gamma$-GTP persisted. We performed liver biopsy to clarify the cause of injury, which revealed panlobular infiltration of inflammatory cells. Most of the infiltrating inflammatory cells were lymphocytes; however, there were a small number of neutrophils, eosinophils, and plasma cells. Portal tracts demonstrated the most severe inflammatory changes, while the intrahepatic bile ducts were intact. There was no confluent necrosis. (Fig. 3a, b). Furthermore, immunohistochemical analyses proved that infiltrating lymphocytes were predominantly CD3-positive (CD3+) and CD8+, and few CD20+ and CD4+ lymphocytes were observed (Fig. 3c-f). Based on these findings, we diagnosed the patient with a case of pembrolizumab-induced hepatitis. We initiated treatment with prednisolone $(0.5 \mathrm{mg} / \mathrm{kg} /$ day) for grade 2 hepatitis, 163 days after the final administration of pembrolizumab. Subsequently, we added azathioprine ( $50 \mathrm{mg} /$ day), 179 days after the final administration of pembrolizumab, due to an increase in the severity of hepatitis (grade 3 ). However, due to the prolonged elevation of AST, ALT, ALP, and $\gamma$-GTP, we increased the dose of azathioprine to 100 $\mathrm{mg} /$ day and prednisolone to $1 \mathrm{mg} / \mathrm{kg} /$ day, 246 and 324 days after the final administration of pembrolizumab, respectively. Thereafter, a decrease in the levels of AST, ALT, ALP, and $\gamma$-GTP was observed. We tapered the dose of prednisolone to $10 \mathrm{mg} /$ day, 400 days after the final administration of pembrolizumab.

\section{Discussion}

To the best our knowledge, this is the first report to reveal the histopathological features of delayed-onset hepatitis as an irAE.

Hepatitis can occur even after the discontinuation of PD-1 inhibitors. Anti-PD-1 antibodies continue to bind to lymphocytes several weeks after the last infusion of PD-1 inhibitors [8]. The aforementioned experimental finding explains the development of irAE after the discontinuation of ICI therapy. Although various delayed-onset irAEs have been reported in the literature, to the best of our knowledge, there was only one case of delayed-onset hepatitis after discontinuation of the ICI therapy [9]. In the aforementioned study on an Australian 


\section{Case Reports in Gastroenterology}

Case Rep Gastroenterol 2020;14:586-592

DOI: 10.1159/000509953 (c) 2020 The Author(s). Published by S. Karger AG, Basel www.karger.com/crg

Kanaoka et al.: Pembrolizumab-Induced Delayed-Onset Hepatitis

patient with advanced melanoma, the authors suspected autoimmune hepatitis as the cause of liver injury, induced by the second-line nivolumab therapy following the first-line ipilimumab therapy [9]. The final diagnosis could not be confirmed because pathological examination was not performed. It is important to consider hepatitis as a possible delayed irAE and monitor changes in liver and biliary tract enzymes even after discontinuation of therapy with ICIs.

We revealed that delayed-onset hepatitis was histopathologically similar to the hepatitis occurring during ICI administration. The constituent inflammatory cells, CD20+/CD3+ and CD4+/CD8+ lymphocyte ratios, and presence or absence of confluent necrosis may be useful to distinguish hepatitis as an irAE from other liver injuries [5]. The characteristic findings of our liver biopsy were as follows: (1) infiltration of CD3+ and CD8+ lymphocytes in panlobular areas, (2) infiltration of few other inflammatory cells, and (3) no confluent necrosis. These findings contradicted those of autoimmune hepatitis or drug-induced liver injury but corresponded to those previously reported in hepatitis that occurred during ICI therapy [4, 5]. Based on these findings, we diagnosed this patient with a case of hepatitis as an irAE of pembrolizumab therapy. Liver biopsy can be useful in the diagnosis of hepatitis even after discontinuation of the ICI therapy.

Unlike our case, in the previous Australian case study, the response to steroid treatment was immediate [9]. The most remarkable difference was the type of serum enzyme elevated (hepatic or biliary tract enzymes). AST and ALT were mainly elevated in the previous case, whereas ALP and $\gamma$-GTP were predominantly elevated in our case. Another previous study on a Japanese patient with pembrolizumab-induced cholestatic liver injury reported a poor and delayed response to steroid treatment [10]. Our case was similar to this Japanese case in that biliary tract enzymes were mainly elevated. Moreover, pathological findings were similar to the exception that bile tract destruction was not observed in our patient on liver biopsy. The chief elevation pattern of biliary tract enzymes suggests cholestatic injuries and may predict poor response to steroid treatment. Administration of prednisolone $(0.5 \mathrm{mg} / \mathrm{kg} /$ day $)$ as well as the addition of azathioprine failed to suppress the deterioration; however, an increase in the dose of prednisolone (up to $1 \mathrm{mg} / \mathrm{kg} /$ day) enabled us to control hepatitis in our case. An increase in the corticosteroid dose might have been most effective in our case; however, azathioprine did not have an immediate effect. In a retrospective French study on 16 patients with hepatitis (grade $\geq 3$ ) as an irAE, six patients showed spontaneous improvement without medications, and four received corticosteroids at a dose of $0.5 \mathrm{mg} / \mathrm{kg} /$ day or less. In this study, only one patient required pulse steroid therapy, with corticosteroid at a dose of $2.5 \mathrm{mg} / \mathrm{kg} /$ day, followed by addition of mycophenolate mofetil as a second immunosuppressive drug. The study reported that ten patients with severe hepatitis showed improvement without corticosteroids or with minimum dose of the drug, and five patients received corticosteroids at a dose of $1 \mathrm{mg} / \mathrm{kg} /$ day [3]. Although the optimal dose of corticosteroids for patients with severe hepatitis remains uncertain, an increase in the dose is one of the options when initial treatment fails to control the condition immediately. The ASCO guideline recommends either mycophenolate mofetil or azathioprine as the second and additional drug option on corticosteroid. However, superiority of either drug remains unknown [6]. Unlike our case, a Japanese melanoma study of nivolumab-induced grade 3 hepatitis reported a good response to azathioprine [11]. In the previous study, $2 \mathrm{mg} / \mathrm{kg} /$ day of methylprednisolone was administered before the initiation treatment with azathioprine. Azathioprine may be one of the options for additional drug therapy in cases of hepatitis not controlled by permissible doses of corticosteroids.

In conclusion, we reported a case of hepatitis as an irAE that developed 2 months after the final administration of pembrolizumab. Pembrolizumab-induced hepatitis was confirmed 


\section{Case Reports in Gastroenterology}

histopathologically. Hepatitis as an irAE should be considered in the differential diagnosis after discontinuation of ICI therapy. Clinicians should not hesitate to perform liver biopsy for confirmation of the diagnosis.

\section{Acknowledgement}

We thank Dr. Shoichi Ihara, Dr. Kanako Nishimatsu, Dr. Saori Ikebe (the Department of Respiratory Medicine, Osaka Police Hospital), Dr. Masahide Oshita, Dr. Satoshi Egawa, Dr. Masanori Miyazaki (the Department of Gastroenterology, Osaka Police Hospital), Dr. Tsunehiro Tanaka, and Dr. Kiyoshi Komuta (the Department of Respiratory Medicine, Daini Osaka Police Hospital) for their contributions to the care and management of this patient.

\section{Statement of Ethics}

The authors have no ethical conflicts to disclose. The patient has given written informed consent to publish this case including publication of images.

\section{Conflict of Interest Statement}

All authors declare no potential conflicts of interest related to the publication of this case report.

\section{Funding Sources}

We declare that the authors received no funds for this research.

\section{Author Contributions}

K.K., K.M., H.O., K.I., and S.M. were involved in treatment of this patient. H.T and H.Y. performed pathological diagnosis of hepatitis. K.K. drafted the report. All authors read and critically reviewed the report. All authors approved the final submitted version.

\section{References}

1 Mok TS, Wu YL, Kudaba I, Kowalski DM, Cho BC, Turna HZ, et al.; KEYNOTE-042 Investigators. Pembrolizumab versus chemotherapy for previously untreated, PD-L1-expressing, locally advanced or metastatic non-small-cell lung cancer (KEYNOTE-042): a randomised, open-label, controlled, phase 3 trial. Lancet. 2019 May;393(10183):1819-30.

2 Wang Y, Zhou S, Yang F, Qi X, Wang X, Guan X, et al. Treatment-Related Adverse Events of PD-1 and PD-L1 Inhibitors in Clinical Trials: A Systematic Review and Meta-analysis. JAMA Oncol. 2019 Jul;5(7):1008-19.

3 De Martin E, Michot JM, Papouin B, Champiat S, Mateus C, Lambotte O, et al. Characterization of liver injury induced by cancer immunotherapy using immune checkpoint inhibitors. J Hepatol. 2018 Jun;68(6):1181-90.

4 Zhang D, Hart J, Ding X, Zhang X, Feely M, Yassan L, et al. Histologic patterns of liver injury induced by antiPD-1 therapy. Gastroenterol Rep (Oxf). 2019 Sep;8(1):50-5. 


\section{Case Reports in Gastroenterology}

\begin{tabular}{l|l}
\hline Case Rep Gastroenterol 2020;14:586-592 \\
\hline DOI: 10.1159/000509953 & $\begin{array}{l}\text { @ 2020 The Author(s). Published by S. Karger AG, Basel } \\
\text { www.karger.com/crg }\end{array}$ \\
\hline
\end{tabular}

5 Zen Y, Yeh MM. Checkpoint inhibitor-induced liver injury: A novel form of liver disease emerging in the era of cancer immunotherapy. Semin Diagn Pathol. 2019 Nov;36(6):434-40.

6 Brahmer JR, Lacchetti C, Schneider BJ, Atkins MB, Brassil KJ, Caterino JM, et al.; National Comprehensive Cancer Network. Management of immune-related adverse events in patients treated with immune checkpoint inhibitor therapy: american society of clinical oncology clinical practice guideline. J Clin Oncol. 2018 Jun;36(17):1714-68.

7 Tian Y, Abu-Sbeih H, Wang Y. Immune Checkpoint Inhibitors-Induced Hepatitis. Adv Exp Med Biol. 2018;995:159-64.

8 Osa A, Uenami T, Koyama S, Fujimoto K, Okuzaki D, Takimoto T, et al. Clinical implications of monitoring nivolumab immunokinetics in non-small cell lung cancer patients. JCI Insight. 2018 Oct;3(19):1-16.

9 Parakh S, Cebon J, Klein O. Delayed Autoimmune Toxicity Occurring Several Months After Cessation of AntiPD-1 Therapy. Oncologist. 2018 Jul;23(7):849-51.

10 Kurokawa K, Hara M, Iwakami SI, Genda T, Iwakami N, Miyashita Y, et al. Cholestatic liver injury induced by pembrolizumab in a patient with lung adenocarcinoma. Intern Med. 2019;58(22):3283-7.

11 Iwamoto K, Ishitsuka Y, Tanaka R, Sekine I, Fujimoto M. Azathioprine combination therapy for steroidrefractory hepatic immune system-related adverse events. Eur J Dermatol. 2017 Jun 1;27(3):301-3.

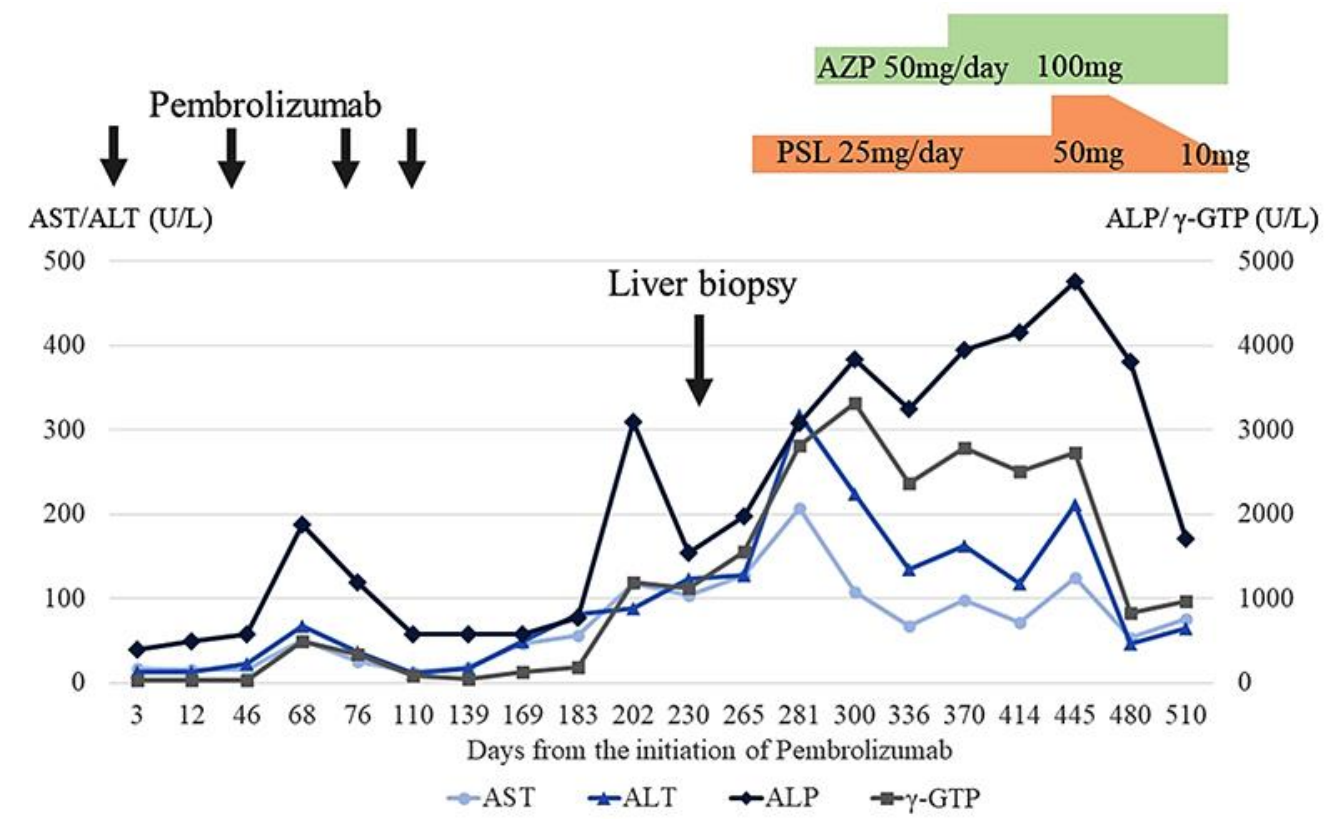

Fig. 1. Clinical course and changes in laboratory data. ALP, alkaline phosphatase; ALT, alanine transaminase; AST, aspartate aminotransferase; AZP, azathioprine; $\gamma$-GTP, $\gamma$-glutamyl transpeptidase; PSL, prednisolone. 


\section{Case Reports in Gastroenterology}

\begin{tabular}{l|l}
\hline Case Rep Gastroenterol 2020;14:586-592 \\
\hline DOI: 10.1159/000509953 & $\begin{array}{l}\text { @ 2020 The Author(s). Published by S. Karger AG, Basel } \\
\text { www.karger.com/crg }\end{array}$ \\
\hline
\end{tabular}

Kanaoka et al.: Pembrolizumab-Induced Delayed-Onset Hepatitis

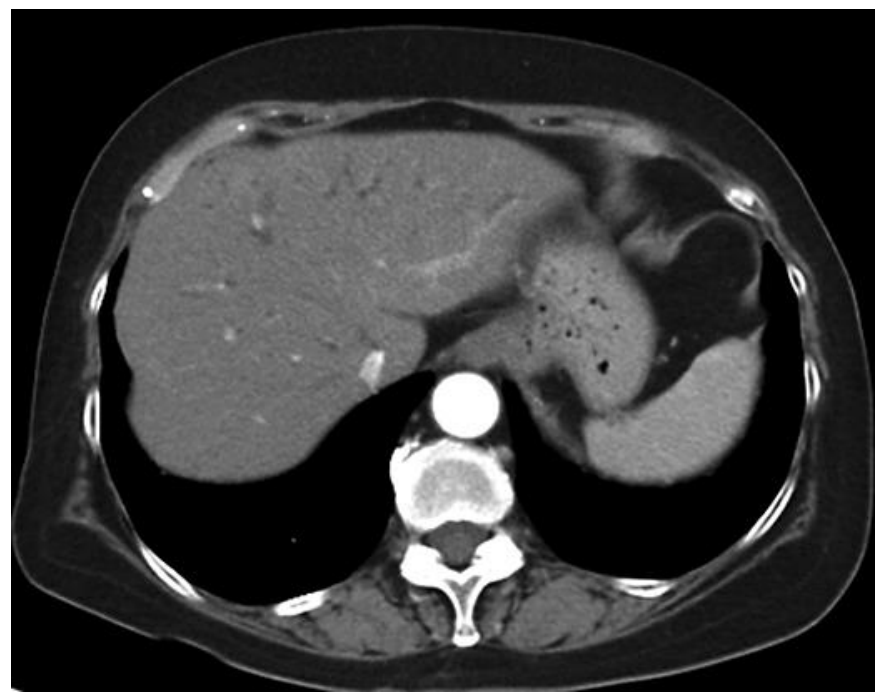

Fig. 2. Abdominal CT image shows mild dilation of the intrahepatic and common bile ducts. Cholecystitis and liver metastasis were not suspected.
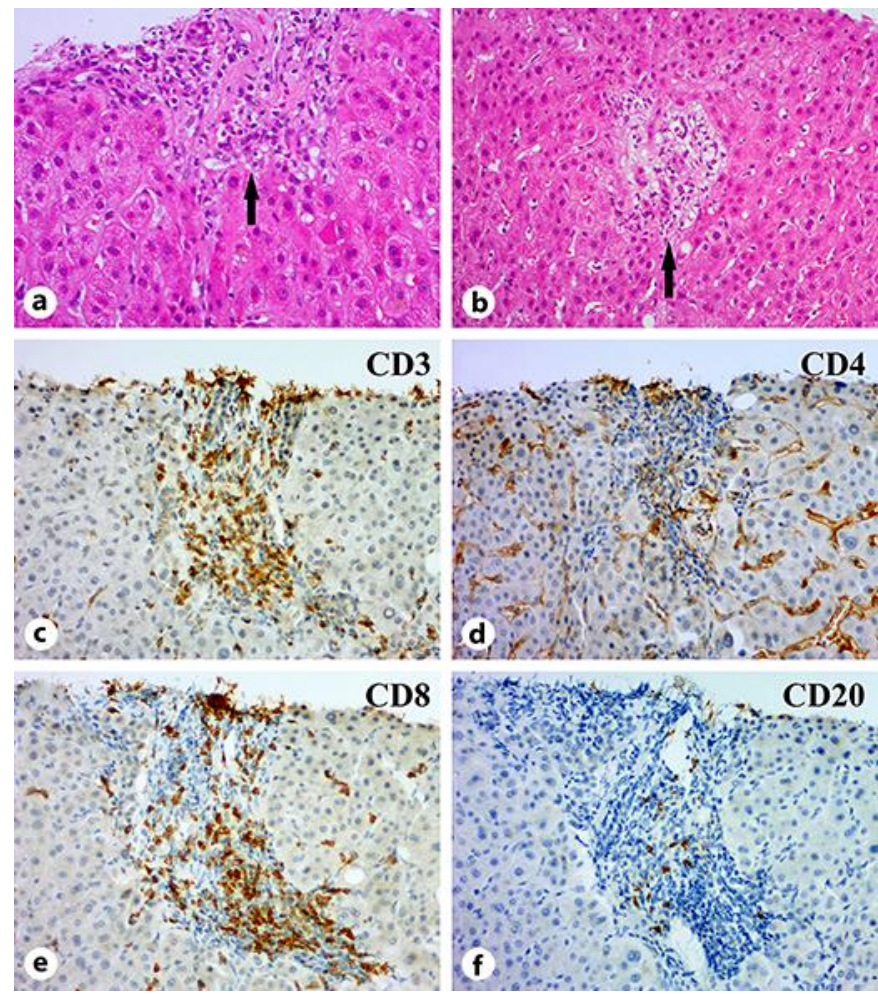

Fig. 3. Hematoxylin and eosin staining of the liver $(\times 100)$. Arrows indicate infiltration of lymphocytes into the portal tract (a) and into the parenchyma (b). Immunostaining $(\times 100)$ for CD3 (c), CD4 (d), CD8 (e), and CD20 (f) lymphocytes. 